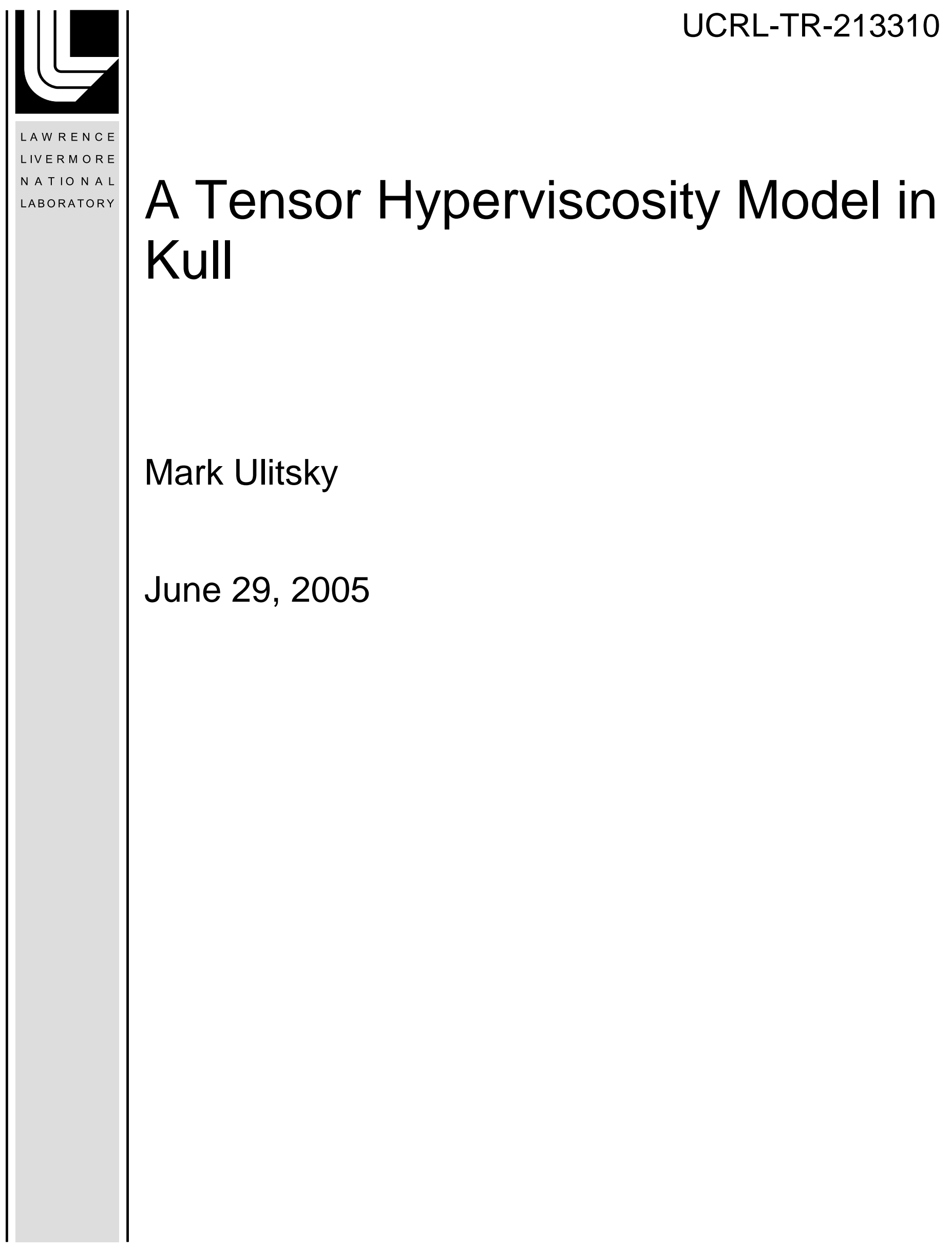


This document was prepared as an account of work sponsored by an agency of the United States Government. Neither the United States Government nor the University of California nor any of their employees, makes any warranty, express or implied, or assumes any legal liability or responsibility for the accuracy, completeness, or usefulness of any information, apparatus, product, or process disclosed, or represents that its use would not infringe privately owned rights. Reference herein to any specific commercial product, process, or service by trade name, trademark, manufacturer, or otherwise, does not necessarily constitute or imply its endorsement, recommendation, or favoring by the United States Government or the University of California. The views and opinions of authors expressed herein do not necessarily state or reflect those of the United States Government or the University of California, and shall not be used for advertising or product endorsement purposes.

This work was performed under the auspices of the U.S. Department of Energy by University of California, Lawrence Livermore National Laboratory under Contract W-7405-Eng-48. 


\section{A Tensor Hyperviscosity Model in Kull}

\section{Mark Ulitsky AX-Division L-023}

A tensor artificial hyper-viscosity model [1] has recently been added to the available list of artificial viscosities that one can chose from when running KULL. This model is based on the theoretical work of A. Cook and B. Cabot, and the numerical results of running the model in the high-order spectral/compact finite difference framework of the Eulerian MIRANDA code. The viscosity model is based on filtering a Laplacian or bi-Laplacian of the strain rate magnitude, and it was desired to investigate whether the formalism that worked so well for the MIRANDA research code could be carried over to an unstructured ALE code like KULL.

The equations solved for a single material, gamma law gas are the standard Euler equations supplemented by an ideal gas eos and an additional stress to account for the artificial viscosity. These equations are given by:

$$
\begin{aligned}
& \frac{d \rho}{d t}=0 \\
& \frac{d \rho u_{i}}{d t}=-\frac{\partial \tau_{i j}}{\partial x_{j}}-\frac{\partial P}{\partial x_{j}} \\
& \frac{d \rho I}{d t}=-P \frac{\partial u_{i}}{\partial x_{i}}-\tau_{i j} S_{i j} \\
& P=(\gamma-1) \rho I \\
& \tau_{i j}=-2 \mu S_{i j}+\left(\frac{2}{3} \mu-\beta\right) \frac{\partial u_{k}}{\partial x_{k}} \delta_{i j} \\
& S_{i j}=\frac{1}{2}\left(\frac{\partial u_{i}}{\partial x_{j}}+\frac{\partial u_{j}}{\partial x_{i}}\right) \\
& \mu=C_{\mu}^{r} \eta_{r}, \quad \eta_{r}=\rho \Delta^{(\mathrm{r}+2) \mid}\left|\nabla^{r} S\right|, r=2,4,6, \ldots \\
& \beta=C_{\beta}^{r} \eta_{r} \\
& S=\sqrt{S_{m n} S_{n m}} \cdot
\end{aligned}
$$

Here, the overbar in the equation for $\eta_{r}$ represents an averaging or filtering process. 
In the MIRANDA code, a truncated Gaussian filter was used to filter the magnitude of the strain rate tensor. In KULL, numerical experimentation on different shock problems has lead to the use of successive arithmetic averages as the desired form for the filtering. What this translates to on a general unstructured mesh is the following. Let's say we want the filtered value at a zone to be influenced by neighbors that are $\sim 3$ zones away in all directions. We compute an initial value of $\eta_{r}$ by taking each zone, looping through all of its nodes, and then storing all of the zones that touch these nodes. For a 2D structured mesh for example, each zone (away from a boundary) would have 9 neighbors from which to compute a value at an interior zone. Note that we consider the original zone to be one of the neighbors. If we now repeat this process two more times, then we are effectively generating a larger region of influence for each zone. Also, while in MIRANDA, $r$ values of 2 or 4 are straightforward to achieve (given the high order difference stencils), in KULL an $r$ value of 2 is probably as high as one can go.

To test the efficacy of the new tensor hyperviscosity, a series of shock test problems has been investigated. These problems include the 1D Shu-Osher problem, the 1D planar Noh problem and the 2D cylindrical Noh problem. Work on the Saltzmann piston problem with a 10 to 1 aspect ratio is still in progress. In all cases, we compare the results of running KULL with the hyperviscosity model to the results from running KULL with the default artificial viscosity model, namely the CSW (Caramana-ShashkovWhalen) edge Q [2] with the recommended coefficients of unity for the linear and quadratic parts. The tensor Q parameters $C_{\mu}^{r}$ and $C_{\beta}^{r}$ were set to .075 and 3.00 respectively.

The initial conditions for the Shu-Osher problem [3] are $\rho=3.857143, p=$ 10.33333 , and $\mathrm{u}_{\mathrm{x}}=2.629369$ for $\mathrm{x}<-4$ and $\rho=1+.2 \sin (5 \mathrm{x}), \mathrm{p}=1$, and $\mathrm{u}_{\mathrm{x}}=0$ for $\mathrm{x}>=-$ 4 , with $\gamma=1.4$. Figure 1 shows the initial conditions for the density field and Figure 2 shows the comparison of the density fields for the two different artificial viscosities at $t=$ 1.8 , with the advection limiter turned off for the CSW Q. Note that in the figure, sgs refers to subgrid scale Q, which is an abbreviated way of referencing the tensor hyperviscosity. While there is no analytic solution for this late time, the near shock wake behavior is remarkably similar for the two different approaches and is also virtually identical to the solution obtained from the 200 zone MIRANDA calculation (not shown). The largest discrepancy occurs in the far wake region, where the CSW Q diffuses the shocklets somewhat more strongly than the tensor Q. Figure 3 shows the same results except that the advection limiter is now turned on for the CSW Q. In this case, the advection limiter keeps the shocklets much sharper and in better agreement with the MIRANDA result.

The KULL calculations had 300 zones, because additional zones needed to be added to the left hand region in order to mimic the correct boundary behavior seen in the Eulerian calculation. Basically, the spatial domain of interest in this problem is from -5 to 5 , and 100 zones were added to the left of $x=-5$ so that the left hand boundary could move at the appropriate constant specified velocity. Figure 4 shows the effect of the filtering process for the tensor Q. For this relatively simple 1D problem, differences are 
easily seen between the case of zero filtering and filtering once when we compute $\eta_{r}$, especially in the near wake region where large overshoots are evident with zero filters. The differences between filtering once, twice, or more are less evident for this problem, but to keep all parameters the same for all the runs, we have decided to set the order of the filtering to 3 . Finally, the length scale $\Delta$ for this problem (needed for the tensor Q model) was chosen to be the width of the zone in the $\mathrm{x}$-direction (i.e., the nodal distance between consecutive nodes on the $\mathrm{x}$-axis).

The planar Noh infinite strength implosion problem [4] has $\rho=1, p=0, \gamma=5 / 3$, and a velocity of -1 for all $\mathrm{x}$, except at $\mathrm{x}=0$, where the velocity is zero. The analytic post shock density is given by $4^{\mathrm{d}}$, where $\mathrm{d}=1$ for a planar geometry, $=2$ for a cylindrical geometry and $=3$ for a spherical geometry, although numerically the wall shock heating has a strong effect on this value as you approach the origin. It should be noted that the addition of an artificial thermal conductivity can greatly improve the agreement with the analytic solution near $\mathrm{x}=0$, however, the exploration and implementation of different thermal conductivities in KULL would be a project in its own right. Thus no effort has been made to improve the near wall behavior.

Figure 5 shows a comparison of the sgs Q with the CSW Q at $\mathrm{t}=.6$ for the case where no advection limiter is used for the CSW Q. For $x>.05$, the results are virtually identical, although some extremely small scale, high-frequency oscillations can be observed in the sgs Q. In MIRANDA, the fundamental variables are passed through a low-pass filter to efficiently remove the high frequency noise introduced by the hyperviscosity. In KULL, it is not clear how to mimic the low pass filter employed by the MIRANDA code. Figure 6 shows the comparison at $t=.6$ for the case where the advection limiter is turned on. Here we see that the main effect of the limiter is to change the behavior of the density profile near the wall. Also, as we lower the cfl value, the density gets closer to 4 and the position of the shock front gets closer to .2 for both the sgs Q and the CSW Q (see Figure 7). The number of zones used for these runs was 100 and the same definition of $\Delta$ was used as in the Shu-Osher problem.

The $2 \mathrm{~d}$ cylindrical Noh problem has the same density and pressure specifications as the 1D planar problem, with the major difference being that it is now the radial velocity that is set to -1 , rather than the $\mathrm{x}$-component of the velocity. Figure 8 shows the gmv mesh used for this convergent shock problem, which consists of a square mesh at the center of the quadrant that fans out into a more polar mesh at larger values of radius. Figures 9 and 10 show the density field (along with the mesh) at $t=.6$ for the CSW Q without and with the advection limiter. For the Shu-Osher problem, turning on the limiter greatly helped prevent the smearing out of the shocks in the far wake region. For the 1D planar Noh problem, turning on or off the limiter only made a difference near the wall boundary. However, for this convergent geometry, turning on the limiter has some dramatically adverse effects. That is, there is a severe mesh imprinting at an angle of 45 degrees and jetting that occurs along this angle as well as along the axes. "Hot spots" are generated by the limiter, which causes the shock front to move to quickly and raises the densities well above their expected values (see the range of values in the colorbar map). For the case without the advection limiter, the shock front is much more symmetric and 
constant, although a large mushroom like structure seems to be developing near the origin. The mesh is also much less distorted than the one is the previous figure. Figure 11 shows the same plot for the tensor Q. For this problem, we compute $\Delta$ from the square root of the zone area. This result shows some mesh imprinting along the 45 degree line and also some mesh distortion near the origin. The results are quantitatively very similar to those presented in Figure 9 in terms of the shock location as well as the values of the maximum densities. Again, the agreement with the analytic solution can be made more accurate by lowering the Courant limit to .2 (the cfl value was .4 for Figures 9-11).

\section{References:}

[1] A. W. Cook and W. H. Cabot, Hyperviscosity for shock turbulent interactions, JCP 203 (2): 379-385, 2005.

[2] E. J. Caramana, M. J. Shashkov, and P. P. Whalen, Formulations of artificial viscosity for multi-dimensional shock wave computations, JCP, 144, 70-97, 1998.

[3] C. W. Shu and S. J. Osher, Efficient implementation of essentially nonoscillatory shock capturing schemes II, JCP, 83, 32-78, 1989.

[4] W. F. Noh, Errors for calculations of strong shocks using an artificial viscosity and an artificial heat flux, JCP, 72, 78-120, 1987. 


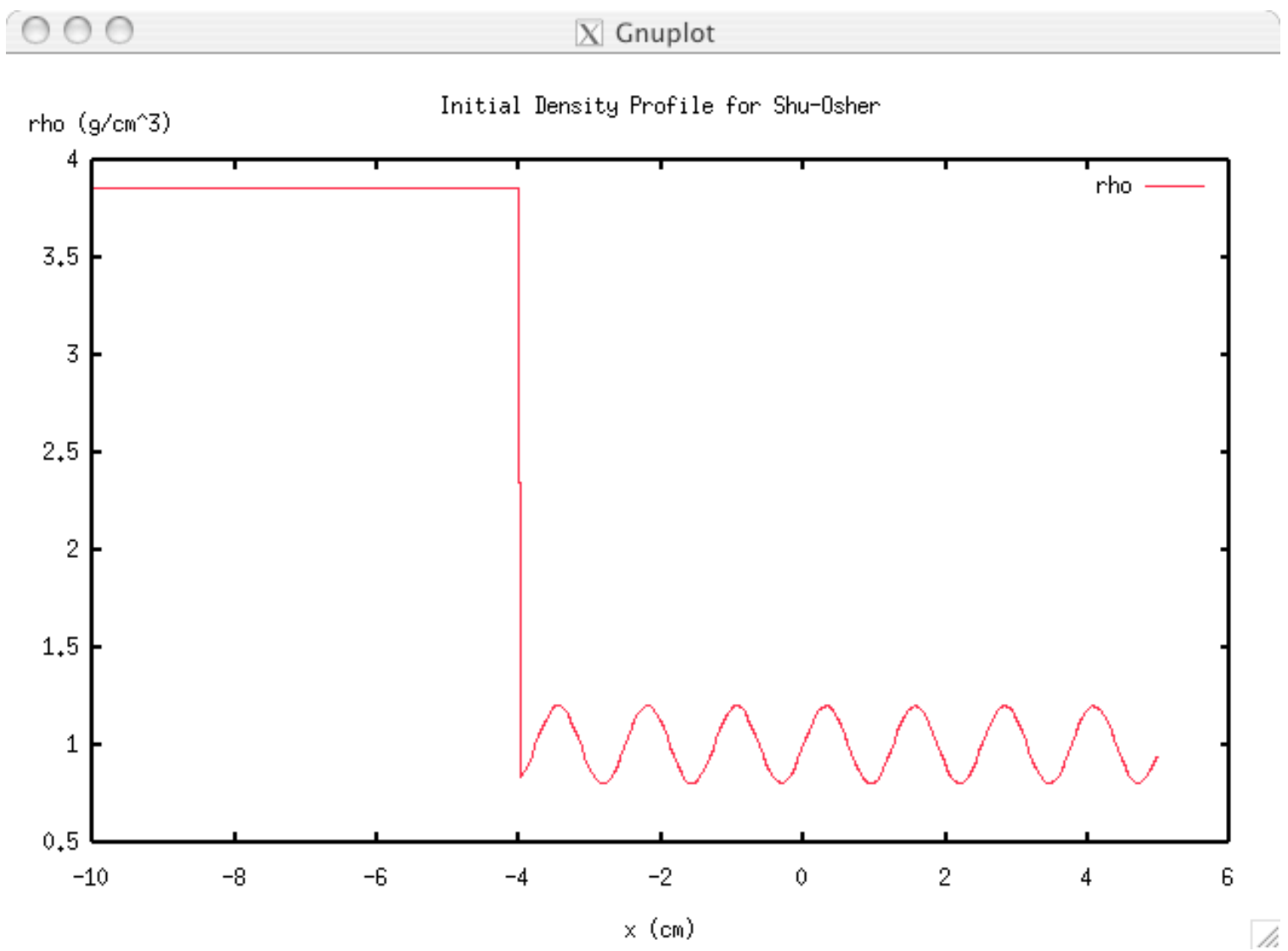

Figure 1 


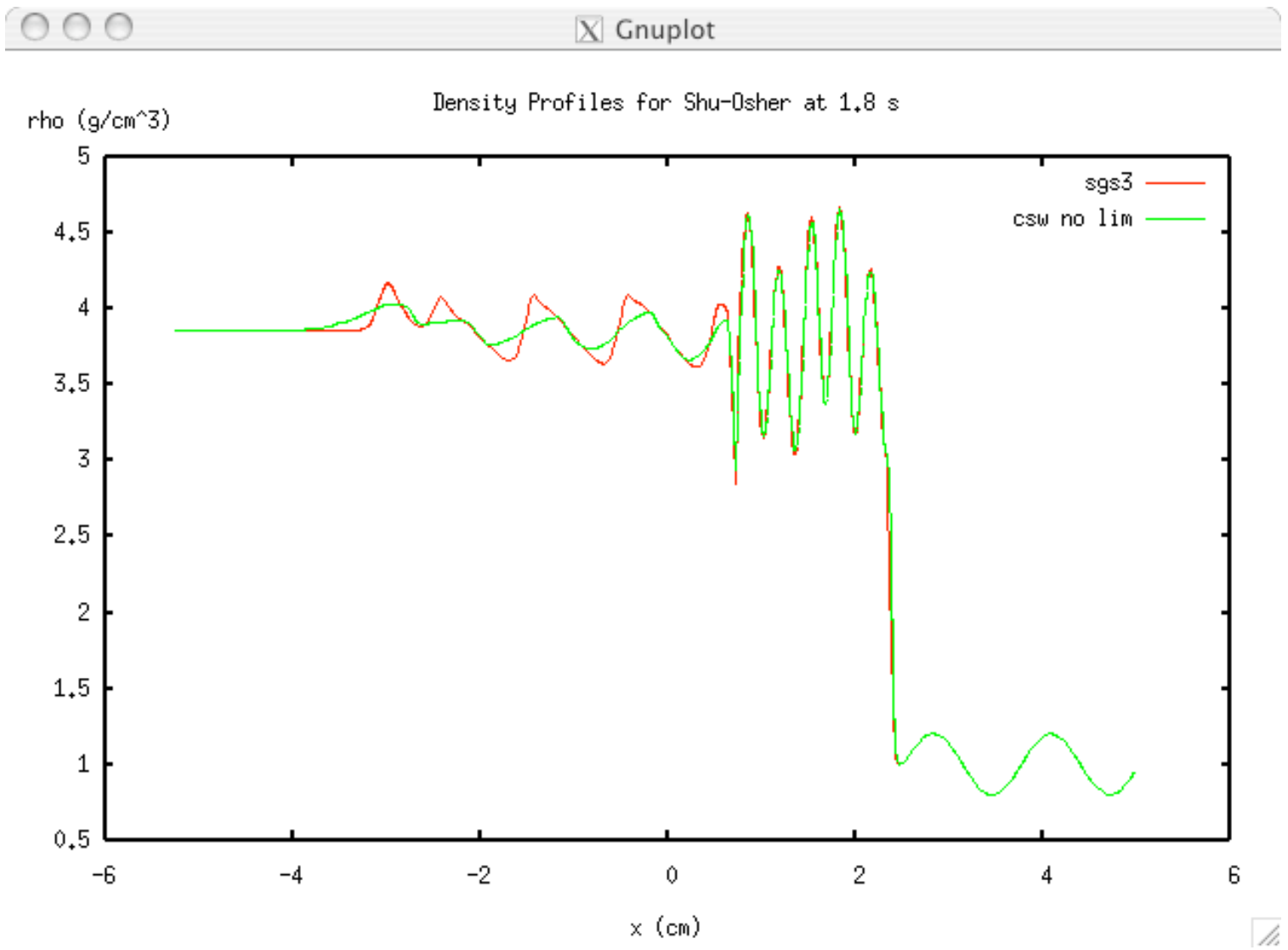

Figure 2 


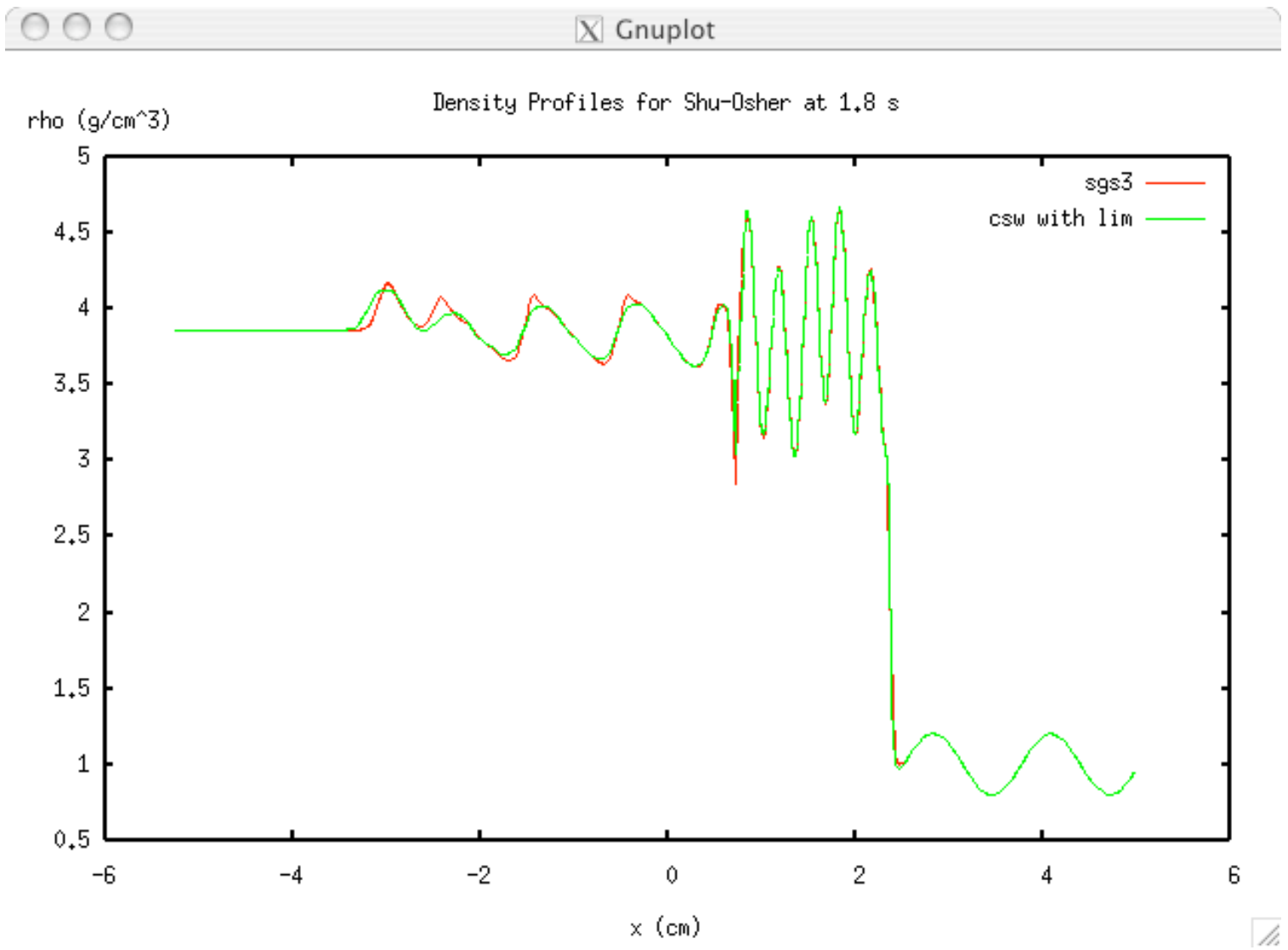

Figure 3 


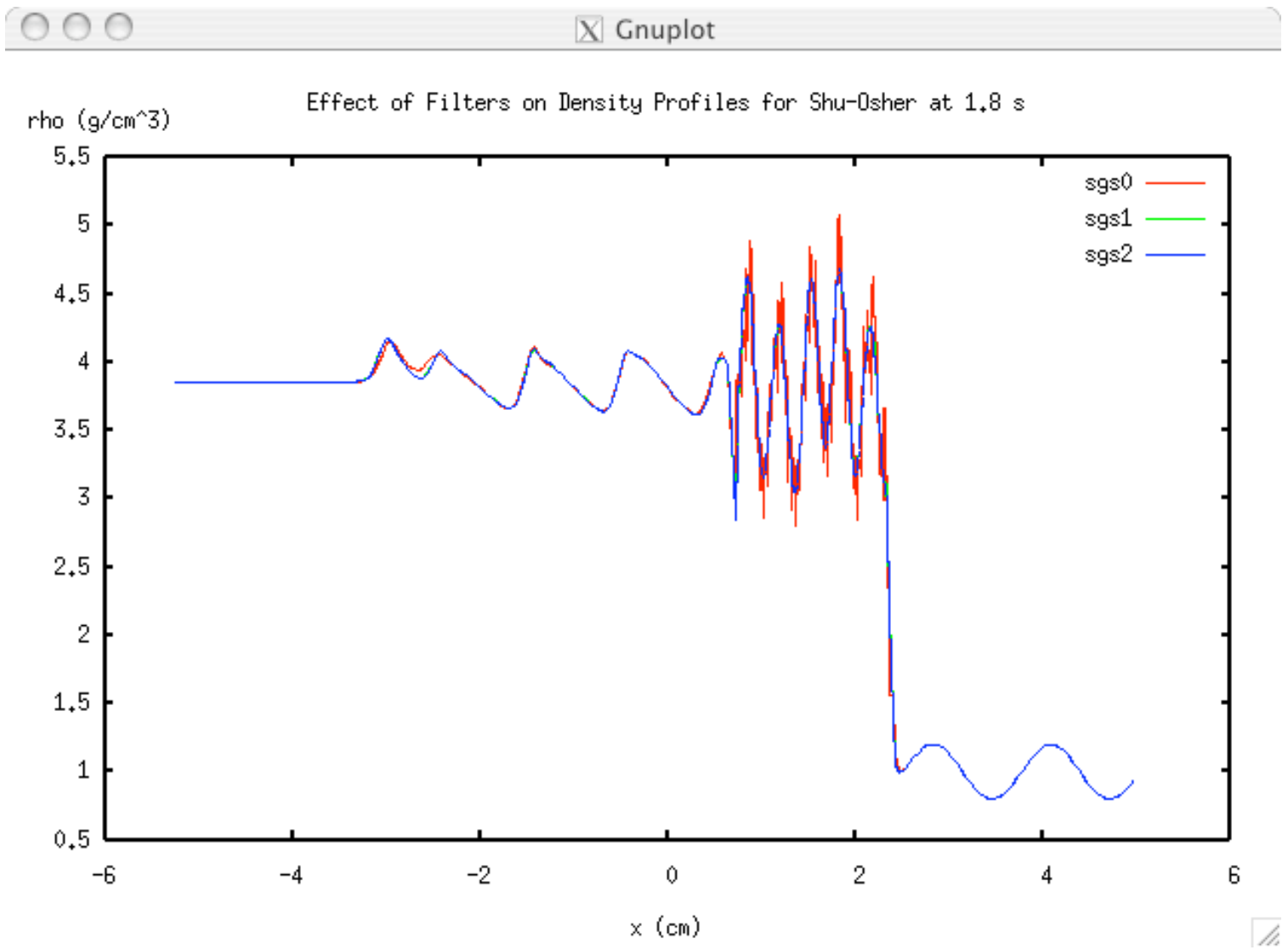

Figure 4 


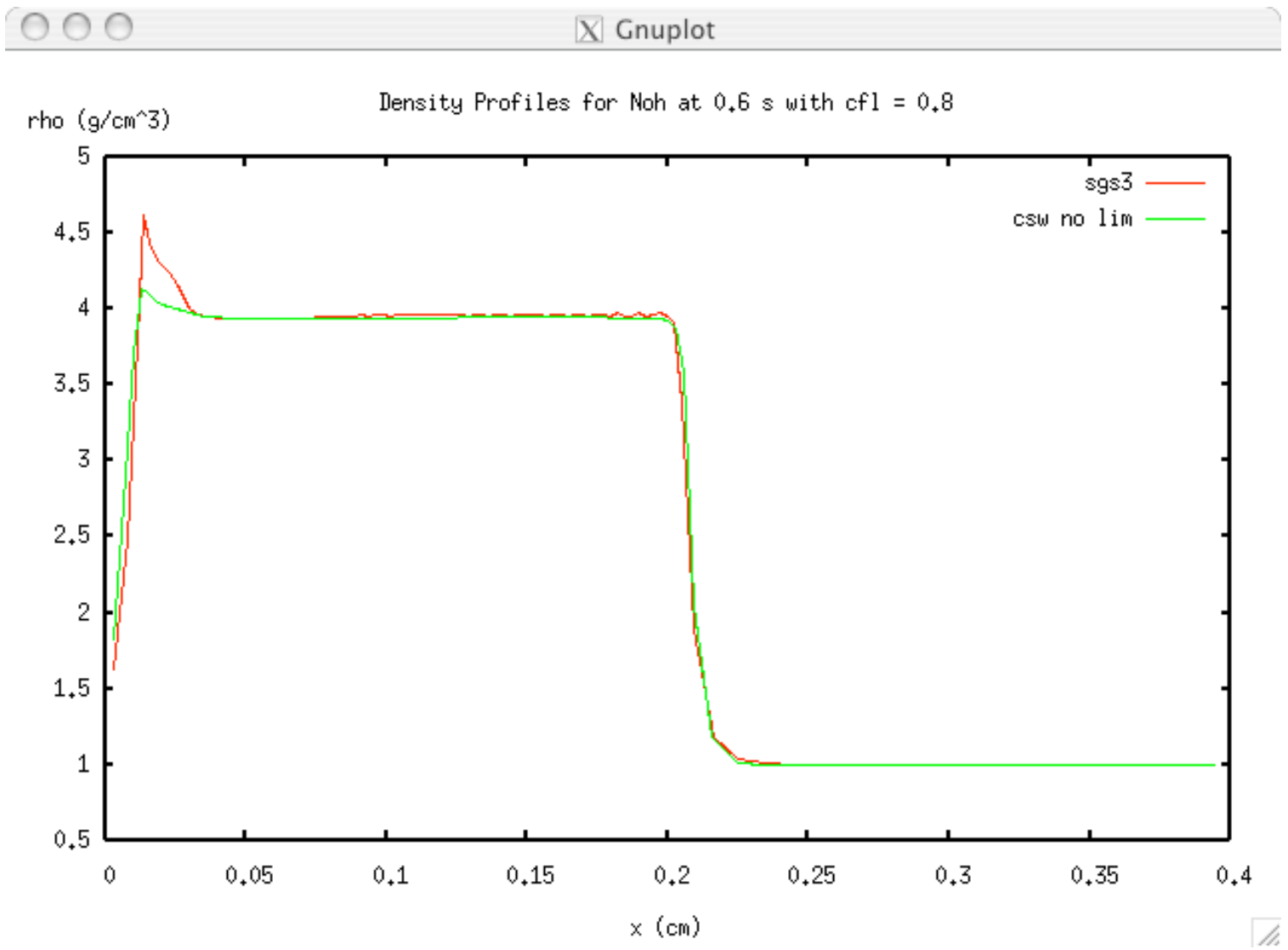

Figure 5 


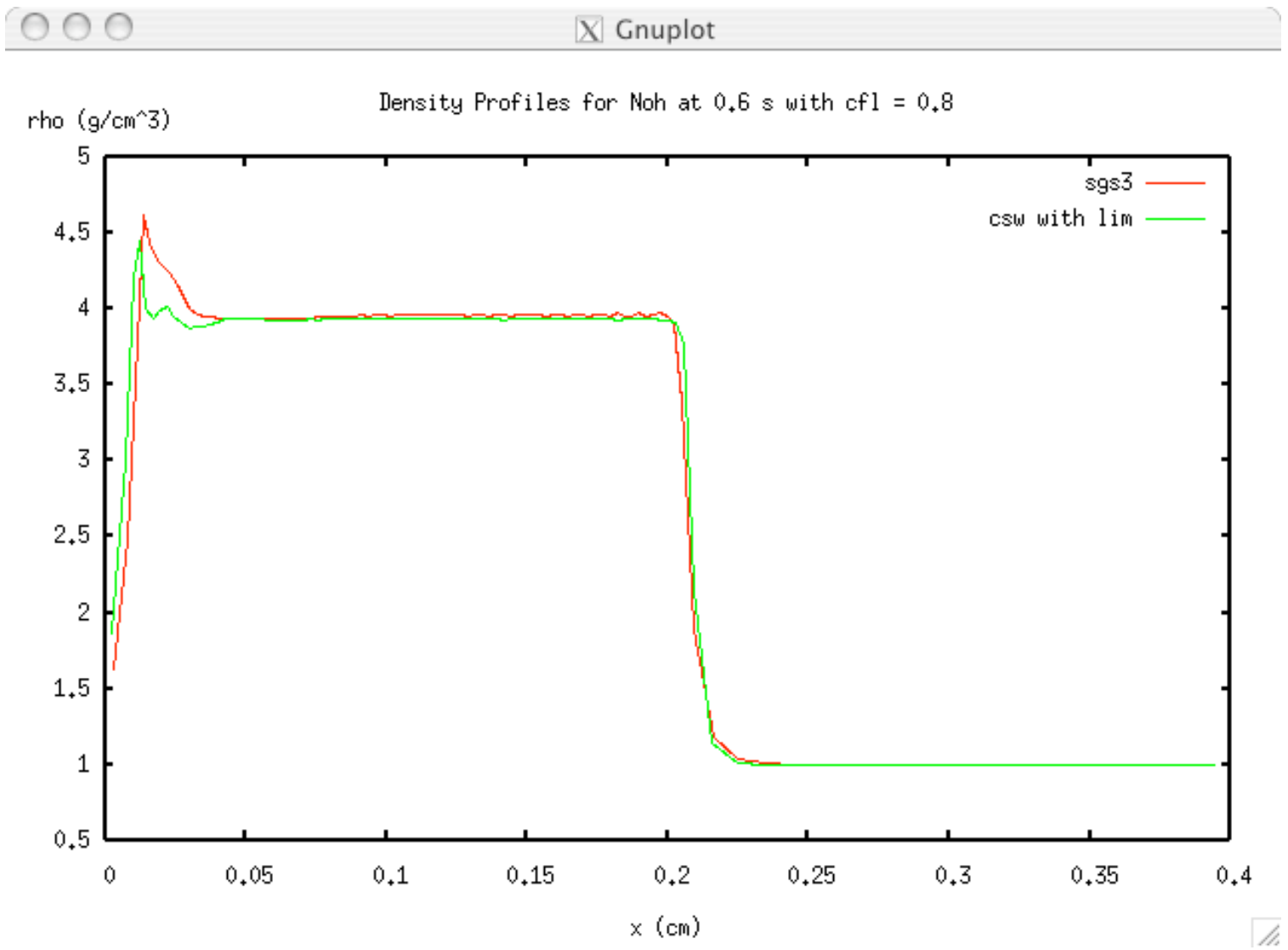

Figure 6 


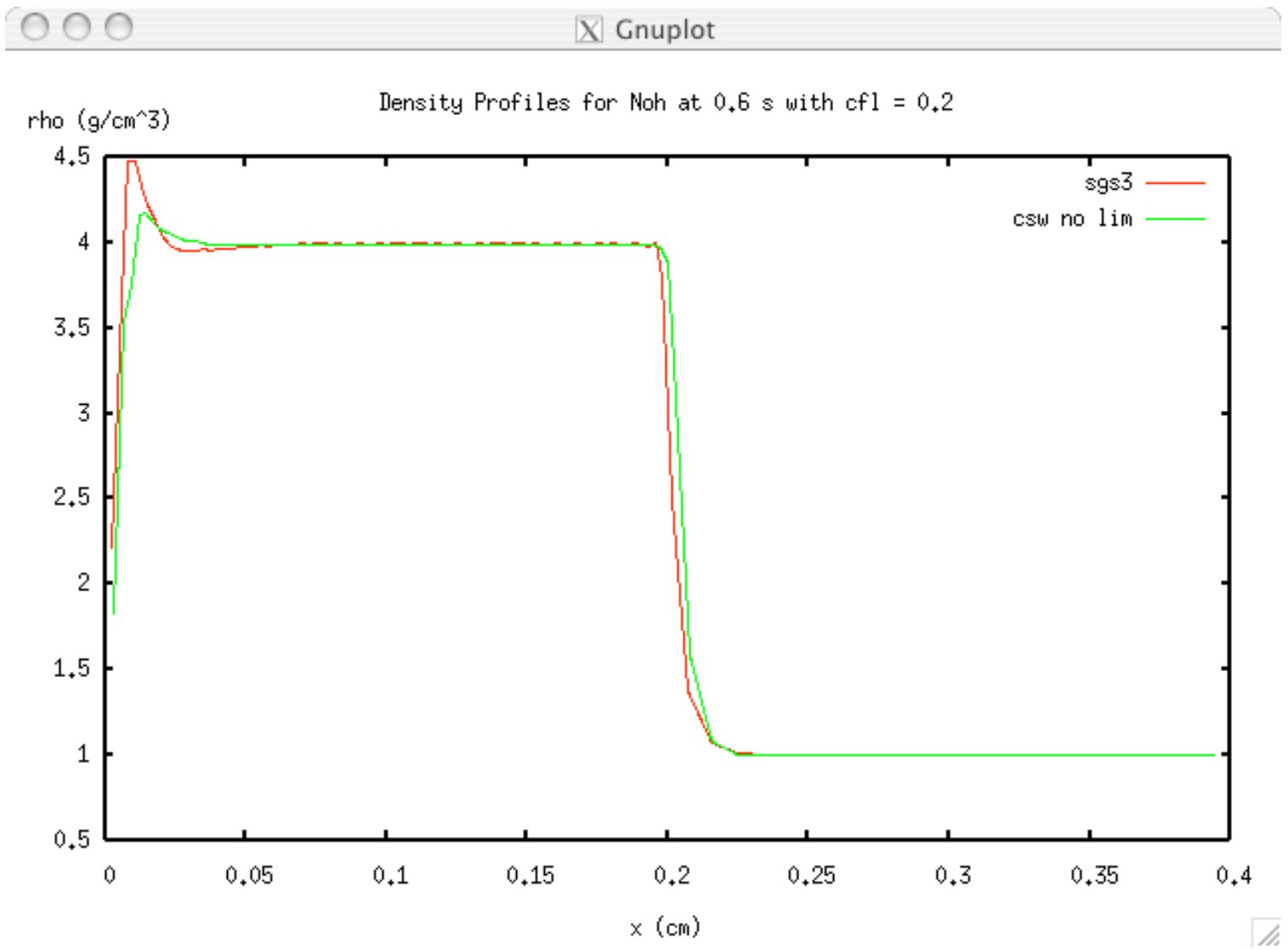

Figure 7 


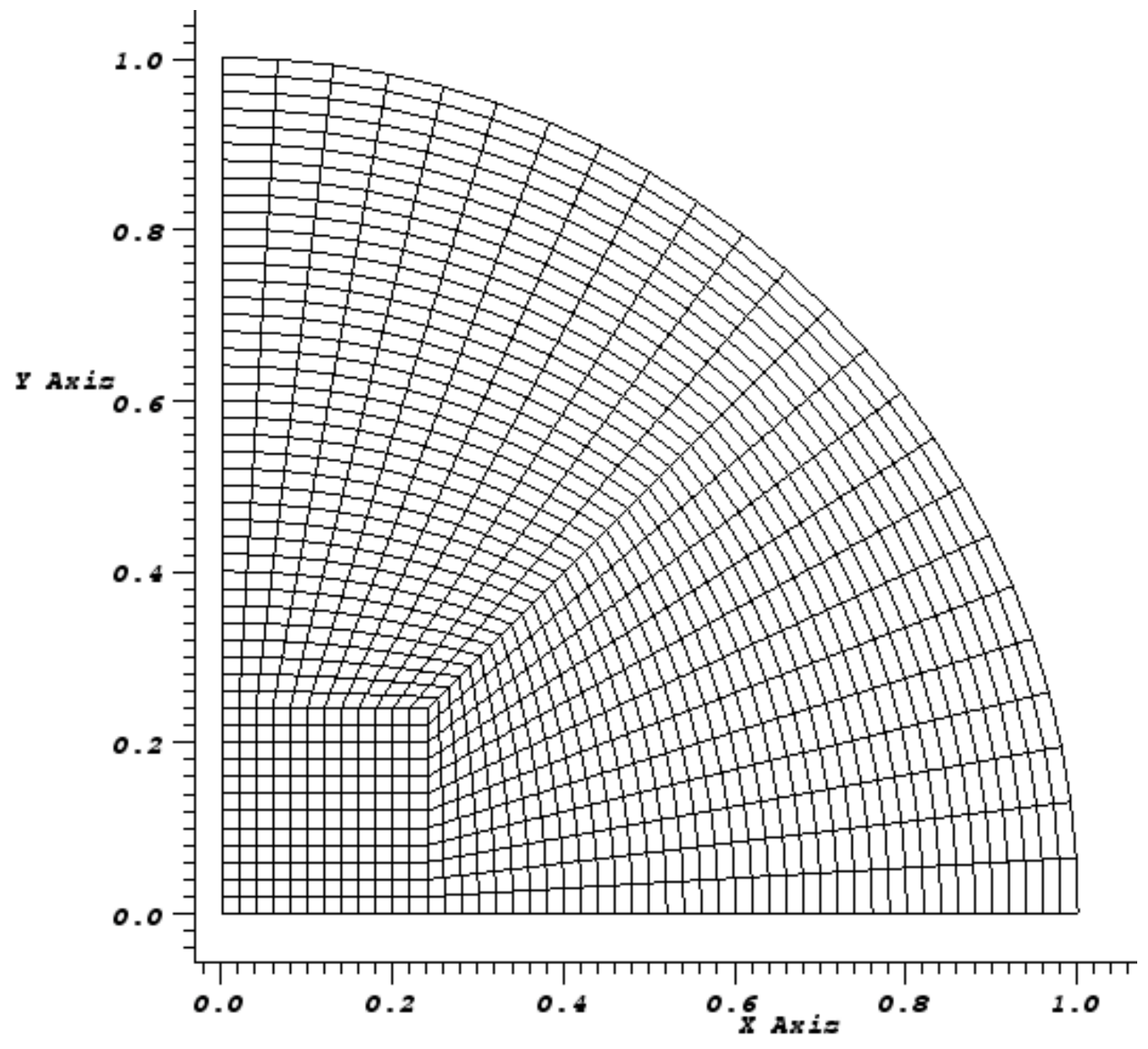

Figure 8 


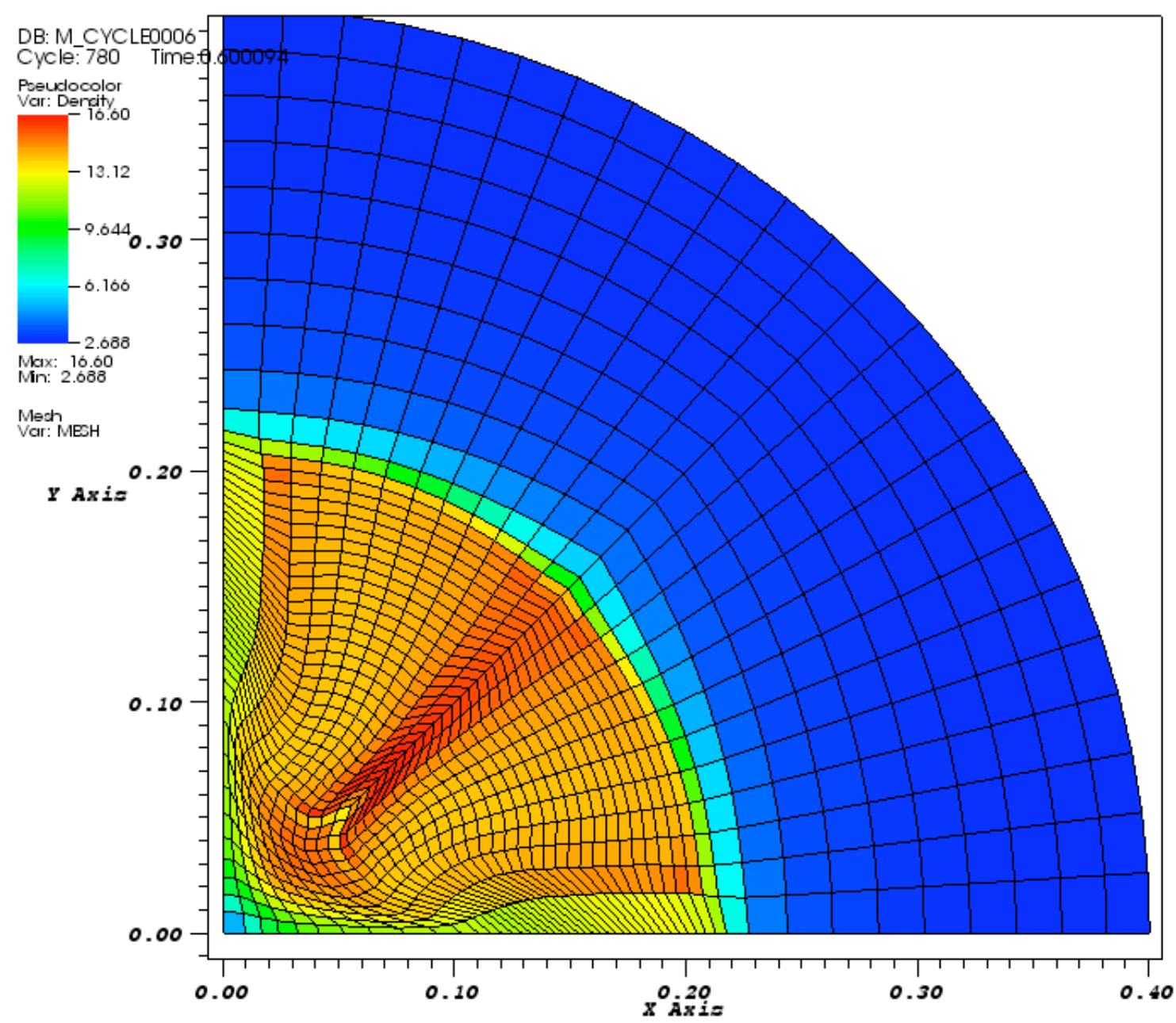

Figure 9 


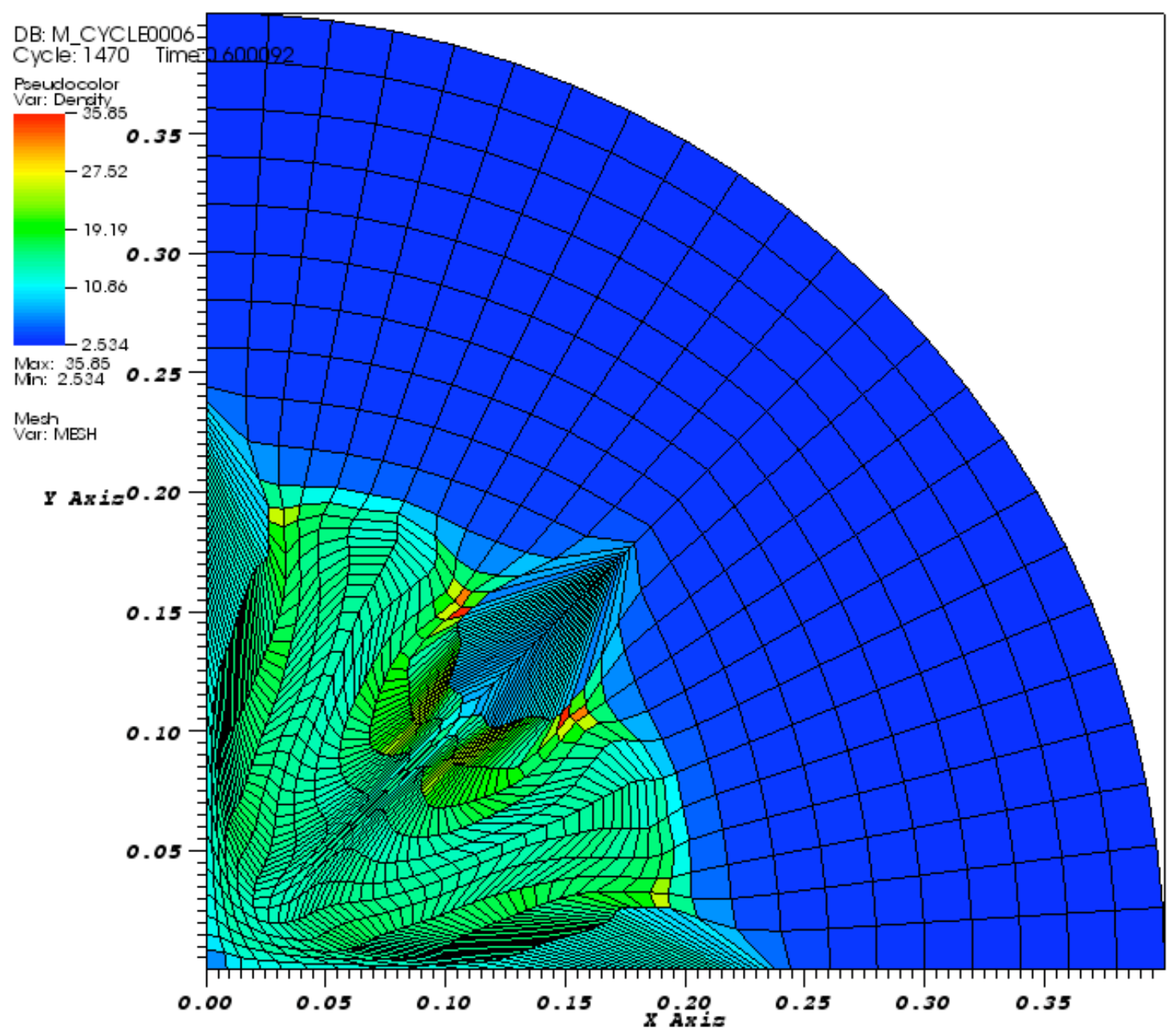

Figure 10 


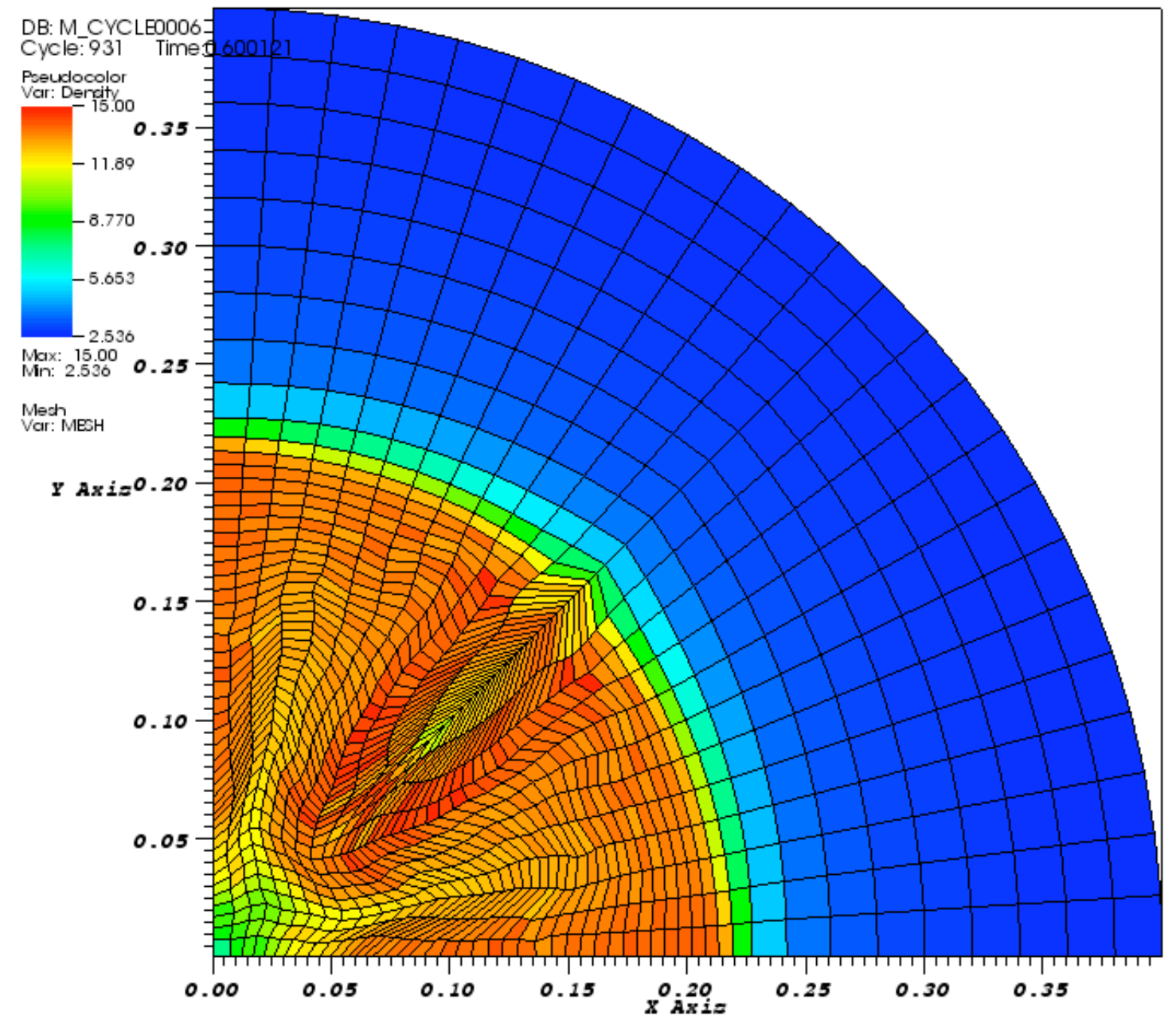

Figure 11 